\title{
Epidemiology, resistant pathogens and main causes of early death of bloodstream infection in patients with hematological malignancies from 2012 to 2019 in a Chinese tertiary hospital
}

\author{
Meng Li \\ Chinese PLA General Hospital \\ Mingmei Du \\ Chinese PLA General Hospital \\ Honghua Li \\ Chinese PLA General Hospital \\ Yunxi Liu ( $\square$ liuyunxi301@qq.com) \\ Chinese PLA General Hospital \\ Daihong Liu ( $\nabla$ daihongrm@163.com ) \\ Chinese PLA General Hospital
}

\section{Research}

Keywords: bloodstream infection, hematological malignancies, Epidemiology

Posted Date: April 20th, 2020

DOI: https://doi.org/10.21203/rs.3.rs-23067/v1

License: (c) (1) This work is licensed under a Creative Commons Attribution 4.0 International License.

Read Full License 


\section{Abstract}

Background: To investigate epidemiology, antibiotic-susceptibility of pathogens, and risk factors for mortality of bloodstream infection (BSI) in patients with hematological malignancies (HMs).

Methods: Single-centre retrospective analysis of BSI episodes in patients with HMs in a Chinese tertiary hospital from 2012 to 2019.

Results: Among 17,796 analyzed admissions, 508 BSI episodes (2.85\%) were identified. Of the 522 isolates, 326 (62.45\%) were Gram-negative bacteria, 173 (33.14\%) were Gram-positive bacteria, and 23 $(4.41 \%)$ were fungi. The incidence of BSI differed significantly among the patients with different $\mathrm{HMs}(\mathrm{P}=$ 0.000): severe aplastic anemia (6.67\%), acute leukemia (6.15\%), myelodysplastic syndrome(3.22\%), multiple myeloma (1.29\%), and lymphoma (1.02\%). Escherichia coli $(30.65 \%, 160 / 522)$ was the most common pathogens, followed by Coagulase-negative staphylococci (CoNS) $(19.35 \%, 101 / 522)$ and Klebsiella pneumonia囚9.96\%, 52/522).

The resistance rates of E. coli, K. pneumonia, P. aeruginosa, and A. baumannii to carbapenems were $6.42 \%, 15.00 \%, 27.78 \%$, and $78.95 \%$, respectively. All the Gram-positive pathogens were susceptible to linezolid, and 3 vancomycin-resistant Enterococcus were isolated. The overall 14-day mortality was $9.84 \%$. The mortality of BSI caused by A. baumannii was $73.86 \%$, while caused by other pathogens was $7.36 \%(\mathrm{p}=0.000)$. A multivariate analysis showed that age $>65$ years, $A$. baumannii and non-remission of the malignancy were independent predictors of 14-day mortality.

Conclusion: Gram-negative bacteria continued to be the most common pathogens causing BSIs in HM patients. An extensive multi-drug resistant baumanni with high mortality rate in HM patients made empirical antimicrobial choice a highly challenging issue.

\section{Background}

Bloodstream infection (BSI) remains a severe complication in patients with hematological malignancies (HMs) entailing high morbidity and mortality. The incidence of BSI in patients with $\mathrm{HMs}$ ranges from $7.16-14.5 \%{ }^{[1-3]}$. Over the past 3 decades, the pathogens of BSI have changed. During the $1980 \mathrm{~s}-1990 \mathrm{~s}$, the pathogens of BSI in patients with HMs were mainly Gram-positive bacteria ${ }^{[4,5]}$. However, the incidence of Gram-negative bacterial infections has risen in recent years ${ }^{[6]}$. Gram-negative pathogens have higher antimicrobial resistance profiles worldwide, especially in China (carbapenem-resistant Klebsiella pneumoniae [CRKP] 20.9\% and carbapenem-resistant Acinetobacter baumannii [CRAB] $70.7 \%$ in 2017) ${ }^{[7]}$. The incidence and pathogens spectrum of BSI may differ by different HMs. Compared to lymphoma or multiple myeloma(MM), the incidence of $B S I$ in patients with acute leukemia (AL) have a much more higher incidence of $B S{ }^{[8]}$. Study showed that in Taiwan BSIs in patients with MM were mainly caused by Gram-negative bacteria ${ }^{[9]}$, whereas in Italy ${ }^{[10]}$ and Finland ${ }^{[11]}$, the BSIs in AL patients were predominantly caused by Gram-positive bacteria. Yet, there have been limited data on BSI in Chinese patients with HMs. 
In this study, we retrospectively analyzed the medical record on BSI in patients with different HMs in a Chinese tertiary hospital from 2012 to 2019 . The epidemiology, antimicrobial susceptibility of pathogens and 14-day mortality of BSI were investigated.

\section{Methods}

\section{Subject information}

The hospital was a tertiary teaching hospital with 4,000 beds in Beijing in north China. There were 125 beds in the Hematology Department, including 20 beds in the hematopoietic stem cell transplantation (HSCT) ward. There were about 2000 patients admitted in Hematology ward every year. The patients admitted in our center between January 1, 2012 and December 31, 2019 were analyzed retrospectively, and predominantly included patients with $A L$, myelodysplastic syndrome (MDS), severe aplastic anemia (sAA), lymphoma, or multiple myeloma (MM). Patients with a hospital stay of $\leq 2$ days were excluded.

\section{Definitions}

A BSI episode was considered present in patients with fever (axillary temperature $>38^{\circ} \mathrm{C}$ for more than 1 h), with or without chill, and with one or more positive blood culturetests ${ }^{[12]}$. Nosocomial BSI was defined as the first positive blood culture obtained $\geq 48 \mathrm{~h}$ after hospital admission and with no evidence of infection at admission. A BSI caused by coagulase-negative staphylococcus (CoNS) or other potential skin contaminants was considered to be significant when the same species grew in at least two blood cultures within a 48-h period ${ }^{[13]}$. Neutropenia was defined as a peripheral blood neutrophil count of $<500$ cells $/ \mathrm{mm}^{3[14]}$.

\section{Surveillance of BSI}

Real-time automatic hospital-wide surveillance of nosocomial infections (NIs) and outbreaks has been established in our hospital since $2012^{[15]}$. In brief, this system automatically records and analyzes microbiological reports, antibiotic usage, imaging reports, fever histories, and other infectious information. The system develops NI prewarning alerts, including BSIs in HM patients. Infection control practitioners then work with the physician-in-charge to confirm the nosocomial BSI, as described in a previous study ${ }^{[15]}$. The information system automatically collects information, such as patient's age, sex, granulocyte count, BSI pathogens and their antimicrobial susceptibility, central venous catheterization, and previous HSCT.

\section{Strain identification and antimicrobial susceptibility testing}

The strains were isolated from the blood samples of patients with BSIs. Only the first detection of a specific pathogen in the same patient was recorded. Blood was cultured with the BacT/ALERT ${ }^{\circledR} 3 D^{\mathrm{TM}}$ system (Becton-Dickinson, Sparks, MD, USA) in the microbiology laboratory. Species were identified and 
then in vitro antibiotic susceptibility was determined with Vitek II (bioMeptibilitys, MD, USA), with the latest breakpoints defined by the Clinical and Laboratory Standards Institute ${ }^{[16]}$.

\section{Statistical analysis}

The $\chi^{2}$ test was used to compare the percentage differences in the univariate analysis. Values of $P<0.05$ were considered statistically significant. Variables found to be significant $(P<0.05)$ in the univariate analysis were tested in a multivariate analysis, which was performed with a stepwise logistic regression model. All statistical analyses were performed with the SPSS 25.0 software (IBM Corp., Armonk, NY, USA).

\section{Results}

\section{Characteristic of BSI in patients with HMs}

There were 17,796 patients with HMs admitted in our center between January 2012 and December 2019. During this 8-year period, $519 \mathrm{BSI}$ episodes were identified. The median age of patients with BSI was 42 years (range 11-94 years) and 335 patients (64.92\%) were man. Table 1 shows baseline patient characteristics.

\section{Incidence of BSI in patients with HMs}

Over the study period, the overall incidence of BSI was $2.92 \%(519 / 17,796)$. The annual incidence of BSI ranged from $2.03 \%$ to $3.54 \%$, which was significantly different $\left(X^{2}=16.126, P=0.024\right)$. The annual incidence of Gram-positive BSI increased significantly $\left(\chi^{2}=31.552 ; P=0.000\right)$. Gram-negative BSIs peaked in 2015 at $2.37 \%$, but fell in 2019 to a frequency similar to that at the start of the study. The annual incidence of Gram-negative BSI and Fungus BSI did not change significantly during the study period $(\chi 2=10.299 ; P=0.172$ and $\chi 2=8.523 ; P=0.289$, respectively). (Figure 1 )

The incidences of BSI according to the different types of HMs were (in descending order): SAA (6.67\%, 15/225), AL (6.15\%, 340/5530), MDS (3.22\%, 23/715), MM (1.29\%, 26/2018), lymphoma (1.02\%, $92 / 9010)$, and other $\mathrm{HMs}(4.03 \%, 12 / 298)$. These incidences differed significantly $\left(X^{2}=346.709, \mathrm{P}=\right.$ $0.000)$.

We divided the patients into two groups according to whether they had undergone HSCT or not. The incidence of BSIs was significantly higher in the HSCT group (10.15\%) than in the non-HSCT group $\left(2.45 \% ; \chi^{2}=161.367 ; P=0.000\right)$. The variations in BSIs in patients with different $\mathrm{HMs}$ are shown in Figure 2.

\section{BSI pathogens and antibiotics susceptibility}

From 2012 to 2019, there were 522 bacteria isolates in 508 patients with BSIs. Of these, Gram-negative bacteria accounted for $62.45 \%$ (326/522), Gram-positive bacteria for $33.14 \%$ (173/522), and fungi for $4.41 \%$ (23/522). The leading Gram-negative bacteria were Escherichia coli (160, 30.65\%), Klebsiella 
pneumonia (52, 9.96\%), and Pseudomonas aeruginosa (41, 7.85\%). Staphylococcus epidermidis (51, 9.77\%), S. hominis (40,7.66\%) and Enterococcus (21, 4.02\%) were the most commonly isolated Grampositive bacteria. 97 CoNS were isolated, although 203 CoNS isolates were considered to be contaminants because only one positive blood culture. The pathogens associated with the different HMs are shown in Table 1.

Isolates of E. coli were highly susceptible to meropenem (90.91\%), amikacin (93.58\%) and imipenem(93.58\%), but exhibited high resistant to ciprofloxacin (84.40\%) in patients with HMs. The resistance rates of $K$. pneumonia and $P$. aeruginosa to carbapenems were $15.00 \%$ and $27.78 \%$, respectively. The susceptibility rates for $K$. pneumoniae to meropenem and amikacin were above $80 \%$. And the susceptibility rates for $P$. aeruginosato to meropenem and amikacin were $69.44 \%$ and $100 \%$, respectively. A. baumannii showed extensive resistance profile, with susceptibility rates to common antibiotics below $50 \%$. Approximately $21.05 \%$ and $27.27 \%$ of $A$. baumannii isolates were susceptible to imipenem and meropenem, respectively (Table 2).

Methicillin-resistant strains accounted for $96.10 \%$ of all CoNS and $33.33 \%$ of S. aureus. All Staphylococcus were susceptible to linezolid. Three vancomycin-resistant Enterococcus isolates (15.79\%) were detected (Table 3).

\section{Outcomes and risk factors for 14-day mortality}

Among the 508 patients with BSIs, 50 (9.84\%) died within 14 days of BSI. The mortality of patients with A. baumannii bacteremia was significantly higher than that of patients infected with other pathogens (73.86\% vs. $\left.7.36 \% \otimes \chi^{2}=326.755, p=0.00\right)$. Among 19 episodes with $A$. baumannii bacteremia in our cohort, 15 patients with CRAB were identified and 14 of them died within 14 days of BSI. A univariate analysis revealed that age $>65$ years, catheter-related infection, the type of pathogen, and the nonremission status of the malignancy correlated with the 14-day mortality, whereas sex, neutropenia, previous HSCT, and HMs subtype did not. The logistic regression analysis results are shown in Table 4. Independent predictors of 14 -day mortality were age $>65$ years (odds ratio [OR]: $2.588 ; 95 \%$ confidence interval [CI]: 1.072-6.249; $\mathrm{P}=0.034$ ), A. baumannii infection (OR: 32.222; 95\% Cl: 8.232-126.126; $\mathrm{P}=$ 0.001 ), and non-remission status of the malignancy (OR: $0.051 ; 95 \% \mathrm{Cl}: 0.007-0.379 ; \mathrm{P}=0.000)($ Table 4).

\section{Discussion}

The overall incidence of BSIs in patients with HMs was about 2.85\% in 2012-2019 in our study, which is significantly lower than that reported in other surveys (about 7.16-14.5\%) ${ }^{[1-3]}$, but higher than the incidence of BSIs in HM patients reported in a retrospective study of a Chinese children's hospital in 2011-2015 (1.04\%) ${ }^{[17]}$. The difference might be attributable to the variation of methods (prospective vs. retrospective) used to monitor BSIs and the different types of HMs or different disease states involved in studies. Another important factor may be the lower submitted blood culture rate in Chinese hospitals than 
in hospitals in developed countries ${ }^{[18]}$. Since 2000 , BSIs caused by Gram-negative bacteria have generally predominated in $\mathrm{HM}$ patients ${ }^{[6]}$. For example, Gram-negative bacteria predominated in studies in $\operatorname{Taiwan}^{[19]}$ and in Italy multicenter survey in HM adult patients in $2009-2012^{[20]}$, accounting for $57.0 \%$ and $52.8 \%$ of BSIs, respectively. In these two studies, E. coli was the most frequently detected Gramnegative bacterium. In our study, Gram-negative bacteria (62.45\%) were the main pathogens of BSIs, and E. coli (30.65\%) was the most frequently detected bacterium, which is consistent with the results of most studies in Tianwan ${ }^{[8,19]}$ and Italy ${ }^{[20]}$. We found that Gram-positive bacteria accounted for $33.14 \%$ of BSIs, but the incidence of it increased significantly from $0.67 \%$ in 2012 to $1.71 \% 2019$ ( $P=0.000)$. 97 strains of CoNS accounted for $18.58 \%$ of BSIs; it was consistent with previous reports in Taiwan $(20.5 \%){ }^{[8]}$ and in Italy $(24.8 \%)^{[20]}$, and in which CoNS were the main Gram-positive bacteria.

Our study found that the incidence of BSIs varied among patients with different types of HMs $(P=0.000)$. The incidence of BSIs was significantly higher in patients with SAA (6.67\%) and AL $(6.15 \%)$ than in those with MM (1.29\%) or lymphoma (1.02\%), consistent with the results reported in other studies. A study by Kara ${ }^{~}{ }^{[2]}$ showed that the incidence of BSIs was $20.2 \%$ in the high-risk group, which contained patients with $\mathrm{AL}$, whereas it was only $10.4 \%$ in the low-risk group,which included patients with non-Hodgkin's lymphoma, $\mathrm{MM}$, or $\mathrm{AA}(\mathrm{P}<0.001)$. According to the report from Taiwan ${ }^{[8]}$, the incidence of $\mathrm{BSIs}$ was highest in patients with $\mathrm{AL}(31.2 \%)$, whereas the rates in patients with myeloma, AA/MDS, and lymphoma were significantly lower $(12.8 \%, 11.1 \%$, and $11.9 \%$, respectively).

In an Italian study of BSIs in patients with AL in 2012-2014 ${ }^{[10]}$, Gram-positive bacteria accounted for $44.8 \%$ and Gram-negative bacteria for $38.3 \%$ of the pathogens involved. More than half BSIs were observed in patients receiving Fluoroquinolone prophylaxis. This was probably the reason for the higher incidence of Gram-positive BSI in comparison with Gram-negative BSI. In another study of BSIs in 357 AML patients with 977 treatment episodes in 2003-2011 in Finland ${ }^{[11]}$, Gram-positive bacteria were most frequently detected (65.7\%). The leading Gram-positive bacteria were CoNS(24.7\%). Contrarily, our results show that although Gram-positive bacteremia increased significantly during this period, the leading pathogens causing BSIs in 5530 admissions with AL were Gram-negative bacteria (64.76\%), with more than half Gram-negative bacteria was E. coli (33.81\%). Gram-negative bacteria have predominated in such patients in most reported studies from Taiwan ${ }^{[8,19,21]}$. These data suggested that the epidemic data for BSI in China was clearly different from that in Western countries.

In the present study, E. coli displayed the highest sensitivity rates to meropenem and amikacin. In contrast, the sensitive rate to ciprofloxacin was significantly lower than that previous reported in $\operatorname{Taiwan}^{[21]}$ (15.6\% vs. $50 \%$ ). The total resistance rates of $K$. pneumonia and $A$. baumannii to imipenem were $15.00 \%$ and $78.95 \%$, respectively. The first CRKP isolated in 2014 in Hematology Department, and it had a marked increase in the studied hospital in recent 5 years. And $A$. baumannii showed extensive antimicrobial resistance profile in the studied hospital. However, the trend of carbapenem-resistant bacteria in our study, was similar to that in the CHINET surveillance from 2005 to 2017 (CRAB ranging from $31 \% \%$ to $70.7 \%$ vs. CRKP ranging from $4.0-20.9 \%)^{[7]}$. 
In this study, $9.84 \%$ of patients with BSIs died within 14 days of infection, which is basically consistent with the results reported in the literature $(12 \%)^{[22]}$. A logistic multivariate regression analysis showed that age $>65$ years, $A$. baumannii and the non-remission of the malignancy were independent risk factors for14-day mortality. Neutropenia was not associated with death from BSIs, which is consistent with results reported in Taiwan ${ }^{[8]}$. This finding is possibly related to the underlying disease and other factors clearly affecting mortality. It is worth noting that among 15 patients with CRAB bacteremia in our cohort, 14 patients died. The mortality of $A$. baumannii bacteremia was higher than that of other pathogens causing BSIs. In China, Tianshui Niu ${ }^{[23]}$ reported that the 28-day mortality rate among 186 patients with A. baumannii BSIs was $45.2 \%$, and the resistance rate of $A$. baumannii to imipenem was $82.6 \%$. The high resistance of $A$. baumanni to carbapenems and its high mortality rate make empirical therapy very challenging.

This study has several limitations. It was a retrospective survey and the data were obtained from an information system. So some information collected might not be incomplete, which might affect the analysis of risk factors. Second, as a single-centre study, it could lead to an inevitable selection bias, and the multivariate logistic analysis might be affected by the sample size. Furthermore, the incidence of BSIs might be underestimated because of the low submission rate of blood culture in the hospital studied.

In conclusion, the incidence of BSIs varied among patients with different HMs. Although Gram-positive bacteremia increased significantly from 2012 to 2019, Gram-negative bacteria continued to be the most common cause of BSIs in each kinds of HM patients. Previous HSCT is a risk factor for BSIs, but did not correlate with 14-day mortality. Age $>65$ years, $A$. baumannii infection, and non-remission status of the malignancy were independent predictors of 14-day mortality. The high resistance of $A$. baumanni to carbapenems and its high mortality rate should be more concerned.

\section{Abbreviations}

BSIs: bloodstream infections

HMs: hematological malignancies

$\mathrm{AL}$ : acute leukemia

sAA: severe aplastic anemia

MDS: myelodysplastic syndrome

HSCT: hematopoietic stem cell transplantation

Nls: nosocomial infections

CLSI: Clinical and Laboratory Standards Institute 


\section{Declarations}

\section{Ethics approval and consent to participate}

This study was approved by the studied hospital institutional review board (S2019-142-02). For this type of study, formal consent is not required.

\section{Consent for publication}

Not applicable.

\section{Availability of data and materials}

The datasets generated during the current study are not publicly available, to avoid disclosure of the individual privacy of the patients. However, they are available from the corresponding author on reasonable request.

\section{Competing interests}

The authors declare that they have no competing interests.

\section{Funding}

This study is funded by the National Key R\&D Program of China (2017YFC0806308). The funders had no role in the design of the study and collection, analysis, and interpretation of data or in writing the manuscript.

\section{Authors' contributions}

M.L. and M.D. collected the data, interpreted the results and wrote the manuscript; H.L. collected and analyzed the data; Y.L. and D.L. designed the study and revised the manuscript. All authors read and approved the final manuscript.

\section{Acknowledgements}

Not applicable.

\section{References}

1. Marín M, Gudiol C, Ardanuy $\mathrm{C}$, et al. Factors influencing mortality in neutropenic patients with haematologic malignancies or solid tumours with bloodstream infection. Clin Microbiol Infect 2015;21(6):583-90. 
2. Kara Ö, Zarakolu P, Aşçioğlu S, et al. Epidemiology and emerging resistance in bacterial bloodstream infections in patients with hematologic malignancies. Infect Dis (Lond) 2015;47(10):686-93.

3. Pagano L, Caira M, Rossi G, et al. A prospective survey of febrile events in hematological malignancies. Ann Hematol 2012;91(5):767-74.

4. Wisplinghoff $\mathrm{H}$, Seifert $\mathrm{H}$, Wenzel RP, Edmond MB. Current trends in the epidemiology of nosocomial bloodstream infections in patients with hematological malignancies and solid neoplasms in hospitals in the United States. Clin Infect Dis 2003;36:1103-10.

5. Zinner SH. Changing epidemiology of infections in patients with neutropenia and cancer: emphasis on gram-positive and resistant bacteria. Clin Infect Dis 1999; 29:490-4.

6. Sara Lo Menzo, Giulia la Martire, Giancarlo Ceccarelli, Mario Venditti. New Insight on Epidemiology and Management of Bacterial Bloodstream Infection in Patients with Hematological Malignancies. Mediterr J Hematol Infect Dis. 2015 ;7(1):e2015044

7. Fupin Hu, Demei Zhu, Fu Wang, and Minggui Wang. Current Status and Trends of Antibacterial Resistance in China. Clin Infect Dis 2018:67 (Suppl 2): S129-34.

8. Chen CY, Tien FM, Sheng WH, Huang SY, Yao M, Tang JL, et al. Clinical and microbiological characteristics of bloodstream infections among patients with haematological malignancies with and without neutropenia at a medical centre in northern Taiwan, 2008-2013. Int J Antimicrob Agents 2017;49(3):272-81.

9. Chun-Teng Huang, Chia-Jen Liu, Po-Shen Ko, et al. Risk factors and characteristics of bloodstream infections in patients with newly diagnosed multiple myeloma. BMC Infectious Diseases (2017) 17:33.

10. Cattaneo C, Zappasodi P, Mancini V, et al. Emerging resistant bacteria strains in bloodstream infections of acute leukaemia patients: results of a prospective study by the Rete Ematologica Lombarda (Rel). Ann Hematol 2016 ;95(12):1955-63.

11. Kolonen A, Sinisalo $M$, Huttunen $R$, et al. Bloodstream infections in acute myeloid leukemia patients treated according to the Finnish Leukemia Group AML-2003 protocol - a prospective nationwide study. Infect Dis (Lond) 2017;49(11-12):799-808.

12. Mermel LA, Allon M, Bouza $E$, et al. Clinical practice guidelines for the diagnosis and management of intravascular catheter related infection: 2009 update by the Infectious Diseases Society of America. Clin Infect Dis 2009;49:1-45.

13. Richter SS, Beekmann SE, Croco JL, et al. Minimizing the workup of blood culture contaminants: implementation and evaluation of a laboratory-based algorithm. J Clin Microbio/ 2002;40(7): 243744.

14. Hughes WT, Armstrong D, Bodey GP, et al. 2002 guidelines for the use of antimicrobial agents in neutropenic patients with cancer. Clin Infect Dis 2002;34:730-51.

15. Du M, Xing Y, Suo J, et al. Real-time automatic hospital-wide surveillance of Nls and outbreaks in a large Chinese tertiary hospital. BMC Med Inform Decis Mak 2014;14:1-9. 
16. Clinical and Laboratory Standards Institute. Performance standards for antimicrobial susceptibility testing; Twenty-third informational supplement. M100-S27. Wayne, Pennsylvania: Clinical and Laboratory Standards Institute, 2017.

17. Jia-Feng Yao, Nan Li, Jin Jiang. Clinical Characteristics of Bloodstream Infections in Pediatric Acute Leukemia: A Single-center Experience with 231 Patients. Chin Med J (Engl) 2017 ;130(17):2076-81.

18. Gao Xiaodong, Hu Bijie, Zhou Chunmei, Cui Yangwen, Shen Yan. Submission rate of blood culture specimens in 72 hospitals in Shanghai. Chin J Nosocomiol 2011;21(12):2602-3.

19. Chen CY, Tang JL, Hsueh PR, Yao M, Huang SY, Chen YC, et al. Trends and antimicrobial resistance of pathogens causing bloodstream infections among febrile neutropenic adults with hematological malignancy. J Formos Med Assoc. 2004 ;103(7):526-32

20. Trecarichi EM, Pagano L, Candoni A, et al. Current epidemiology and antimicrobial resistance data for bacterial bloodstream infections in patients with hematologic malignancies: an Italian multicentre prospective survey. Clin Microbiol Infect 2015;21(4):337-43.

21. Chen CY, Tsay W, Tang JL, Tien HF, Chen YC, Chang SC, et al. Epidemiology of bloodstream infections in patients with haematological malignancies with and without neutropenia. Epidemiol Infect $2010 ; 138(7): 1044-51$.

22. Marin M, Gudiol C, Ardanuy C, et al. Bloodstream infections in neutropenic patients with cancer: differences between patients with haematological malignancies and solid tumours. J Infect 2014;69(5):417-23.

23. Tianshui Niu, TingTing Xiao, Li hua Guo, et al. Retrospective comparative analysis of risk factors and outcomes in patients with carbapenem resistant Acinetobacter baumannii bloodstream infections: cefoperazone-sulbactam associated with resistance and tigecycline increased the mortality. Infect Drug Resist2018; 11: 2021-30.

\section{Tables}

Table 1 Distribution of pathogens causing BSIs 


\begin{tabular}{|c|c|c|c|c|c|}
\hline \multirow[t]{2}{*}{ Pathogens } & \multicolumn{2}{|c|}{ Leukemia AA/MDS } & \multirow[t]{2}{*}{ Myeloma (n=94) } & \multirow[t]{2}{*}{$(n=27)$} & \multirow{2}{*}{$\begin{array}{l}\text { Total } \\
(n=522)\end{array}$} \\
\hline & $(n=349) \quad(n=39$ & & & & \\
\hline G+ & 105(30.09) & $10(25.64)$ & $42(44.68)$ & $11(40.74)$ & $173(33.14)$ \\
\hline Staphylococcus epidermidis & $35(10.03)$ & $3(7.69)$ & $9(9.57)$ & $1(3.70)$ & $51(9.77)$ \\
\hline Staphylococcus hominis & $23(6.59)$ & $3(7.69)$ & $9(9.57)$ & $4(14.81)$ & $40(7.66)$ \\
\hline Enterococcus & $15(4.30)$ & $2(5.13)$ & $4(4.25)$ & $0(0.00)$ & $21(4.02)$ \\
\hline Staphylococcus aureus & $6(1.72)$ & $1(2.56)$ & $7(7.45)$ & $3(11.11)$ & $17(3.26)$ \\
\hline Others & $26(7.45)$ & $1(2.56)$ & 13(13.83) & $4(14.81)$ & $44(8.43)$ \\
\hline G- & $226(64.76)$ & 28(71.79) & $51(54.26)$ & $12(44.44)$ & $326(62.45)$ \\
\hline Escherichia coli & $118(33.81)$ & $9(23.07)$ & $25(26.60)$ & $6(22.22)$ & $160(30.65)$ \\
\hline Klebsiella pneumoniae & $34(9.74)$ & $10(25.64)$ & $5(5.32)$ & $2(7.41)$ & $52(9.96)$ \\
\hline Pseudomonas aeruginosa & $28(8.02)$ & $5(12.82)$ & $4(4.26)$ & $2(7.41)$ & $41(7.85)$ \\
\hline Acinetobacter baumannii & $10(2.87)$ & $5(12.82)$ & $5(5.32)$ & $0(0.00)$ & $19(3.64)$ \\
\hline Stenotrophomonas maltophilia & $9(2.58)$ & $0(0.00)$ & $0(0.00)$ & $0(0.00)$ & $13(2.49)$ \\
\hline Others & $27(7.74)$ & $1(2.56)$ & $12(12.77)$ & $2(7.41)$ & $41(7.85)$ \\
\hline Fungus & $18(5.16)$ & $1(2.56)$ & $1(1.06)$ & $3(11.11)$ & $23(4.41)$ \\
\hline
\end{tabular}

Table 2 Antimicrobial susceptibility profiles of Gram-negative bacteria 


\begin{tabular}{|c|c|c|c|c|}
\hline & Escherichia coli & Pseudomonas aeruginosa & Klebsiella pneumoniae & Acinetobacter baumannii \\
\hline Cefepime & $62.39 \square 68 / 109 \square$ & $77.78 \square 28 / 36 \square$ & $77.50 \square 31 / 40 \square$ & $21.05 \square 4 / 19 \square$ \\
\hline Ceftriaxone & $35.29 \llbracket 36 / 102 \square$ & $0.00 \square 0 / 31 \square$ & $61.76 \square 21 / 34 \square$ & $0.00 \square 0 / 9 \square$ \\
\hline Ceftazidime & $65.74 \square 71 / 108 \square$ & $75.00 \square 27 / 36 \square$ & $70.00 \square 28 / 40 \square$ & $36.84 \square 7 / 19 \square$ \\
\hline Piperacillin tazobactam & 87.01ロ67/77ロ & $88.24 \square 30 / 34 \square$ & $83.33 \llbracket 25 / 30 \square$ & $44.44 \square 4 / 9 \square$ \\
\hline Imipenem & $93.58 \square 102 / 109 \square$ & $72.22 \llbracket 26 / 36 \square$ & $85.00 \square 34 / 40 \square$ & $21.05 \square 4 / 19 \square$ \\
\hline Meropenem & $90.91 \square 30 / 33 \square$ & $69.44 \square 25 / 36 \square$ & $88.88 \square 16 / 18 \square$ & $27.27 \square 3 / 11 \square$ \\
\hline Ciprofloxacin & $15.60 \square 17 / 109 \square$ & $94.44 \square 34 / 36 \square$ & $70.00 \square 28 / 40 \square$ & $15.79 \square 3 / 19 \square$ \\
\hline Amikacin & $93.58 \square 102 / 109 \square$ & $100.00 \square 36 / 36 \square$ & $92.50 \square 37 / 40 \square$ & $50.00 \square 3 / 6 \square$ \\
\hline Gentamycin & $46.08 \square 47 / 102 \square$ & $96.97 \square 32 / 33 \square$ & $76.47 \square 26 / 34 \square$ & $44.44 \square 4 / 9 \square$ \\
\hline Nitrofurantoin & $71.74 \square 66 / 92 \square$ & $0.00 \square 0 / 31 \square$ & $7.14 \square 2 / 28 \square$ & $0.00 \square 0 / 8 \square$ \\
\hline SMZ-TMP & $34.83 \sqcap 31 / 89 \square$ & $0.00 \square 0 / 31 \square$ & $51.61 \square 16 / 31 \square$ & $33.33 \square 3 / 9 \square$ \\
\hline
\end{tabular}

Table 3 Antimicrobial susceptibility profiles of Gram-positive bacteria 


\begin{tabular}{|c|c|c|c|c|}
\hline & Staphylococcus epidermidis & Staphylococcus hominis & Staphylococcus aureus & Enterococcus \\
\hline Oxacillin & $2.50 \square 1 / 40 \square$ & $5.41 \square 2 / 37 \square$ & $66.67 \square 8 / 12 \square$ & NA \\
\hline Ofloxacin & $22.50 \square 9 / 40 \square$ & $21.05 \square 8 / 38 \square$ & $72.73 \llbracket 8 / 11 \square$ & $0.00 \square 0 / 15 \square$ \\
\hline Moxifloxacin & $22.50 \square 9 / 40 \square$ & $21.05 \square 8 / 38 \square$ & $75.00 \square 9 / 12 \square$ & $0.00 \square 0 / 19 \square$ \\
\hline Ciprofloxacin & $22.50 \square 9 / 40 \square$ & $21.05 \square 8 / 38 \square$ & $75.00 \square 9 / 12 \square$ & $0.00 \square 0 / 15 \square$ \\
\hline Gentamycin & $64.29 \square 27 / 42 \square$ & $94.74 \square 36 / 38 \square$ & $64.29 \llbracket 9 / 14 \square$ & $12.50 \square 1 / 8 \square$ \\
\hline Erythromycin & $19.05 \square 8 / 42 \square$ & $7.89 \square 3 / 38 \square$ & $35.71 \square 5 / 14 \square$ & $13.33 \llbracket 2 / 15 \square$ \\
\hline Tetracycline & $77.50 \square 31 / 40 \square$ & $44.74 \square 17 / 38 \square$ & $91.67 \square 11 / 12 \square$ & $40.00 \square 6 / 15 \square$ \\
\hline Vancomycin & $100.00 \square 42 / 42 \square$ & $100.00 \square 38 / 38 \square$ & $100.00 \square 11 / 11 \square$ & $84.21 \square 16 / 19 \square$ \\
\hline Linezolid & $100 \square 40 / 40 \square$ & $100.00 \square 38 / 38 \square$ & $100.00 \square 11 / 11 \square$ & $100.00 \square 15 / 15 \square$ \\
\hline SMZ-TMP & $17.50 \square 7 / 40 \square$ & $18.42 \square 7 / 38 \square$ & $54.55 \square 6 / 11 \square$ & NA \\
\hline Nitrofurantoin & $100.00 \square 26 / 26 \square$ & 97.37ロ37/38凸 & $100.00 \square 11 / 11 \square$ & $12.50 \square 2 / 16 \square$ \\
\hline
\end{tabular}

Table 4 Univariate and multivariate analyses of 14-day death-related risk factors in patients with BSIs 


\begin{tabular}{|c|c|c|c|c|c|c|}
\hline Prognostic factor & \multicolumn{2}{|c|}{ No. of patients } & \multirow{2}{*}{$\begin{array}{l}\text { Univariate } \\
\qquad X^{2}\end{array}$} & \multirow{2}{*}{$\begin{array}{l}\text { analysis } \\
\text { P Value }\end{array}$} & \multicolumn{2}{|c|}{ Multivariate analysis } \\
\hline & Survival & Death(\%) & & & OR $(95 \% \mathrm{CI})(95 \% \mathrm{CI})$ & $P$ Value \\
\hline Age (years) & & & $X^{2}=11.247$ & $P=0.001$ & $2.588(1.072-6.249)$ & $P=0.034$ \\
\hline$>65$ & 32 & $11(25.58)$ & & & & \\
\hline$\leq 65$ & 426 & $39(8.39)$ & & & & \\
\hline Gender & & & $X^{2}=0.026$ & $P=0.871$ & & \\
\hline Female & 161 & $17(9.55)$ & & & & \\
\hline Male & 297 & $33(10.00)$ & & & & \\
\hline Neutropenia & & & $X^{2}=0.004$ & $P=0.950$ & & \\
\hline Yes & 359 & $39(9.80)$ & & & & \\
\hline No & 99 & $11(10.00)$ & & & & \\
\hline Catheter-related infection & & & $X^{2}=5.106$ & $P=0.024$ & $3.627(0.746-17.636)$ & $P=0.110$ \\
\hline Yes & 73 & $2(2.67)$ & & & & \\
\hline No & 385 & 48(11.09) & & & & \\
\hline HMs subtype & & & $X^{2}=8.060$ & $P=0.153$ & & \\
\hline $\mathrm{AL}$ & 312 & $28(8.24)$ & & & & \\
\hline sAA & 10 & $5(33.33)$ & & & & \\
\hline MDS & 20 & $3(13.04)$ & & & & \\
\hline Lymphoma & 83 & $9(9.78)$ & & & & \\
\hline $\mathrm{MM}$ & 23 & $3(11.54)$ & & & & \\
\hline Others & 10 & $2(16.67)$ & & & & \\
\hline Types of pathogens & & & $X^{2}=30.888$ & $P=0.000$ & & $P=0.000$ \\
\hline
\end{tabular}




\begin{tabular}{|c|c|c|c|c|c|c|}
\hline Gram-positive & 166 & $8(4.82)$ & & & - & - \\
\hline Gram-negative (e.x. AB ) & 281 & $25(8.62)$ & & & $1.450(0.642-3.274)$ & $P=0.315$ \\
\hline $\mathrm{AB}$ & 5 & 14(70.59) & & & $35.483(9.441-133.352)$ & $P=0.000$ \\
\hline Fungus & 20 & $3(9.52)$ & & & $2.494(0.667-9.325)$ & $P=0.630$ \\
\hline HSCT & & & $\mathrm{X}^{2}=4.684$ & $\mathrm{P}=0.096$ & & \\
\hline Allogeneic HSCT & 91 & $16(14.95)$ & & & & \\
\hline Autologous HSCT & 26 & $1(3.70)$ & & & & \\
\hline \multicolumn{7}{|l|}{ No } \\
\hline No & 341 & $33(8.82)$ & & & & \\
\hline Disease status & & & $X^{2}=22.811$ & $P=0.000$ & $0.051(0.007-0.379)$ & $P=0.004$ \\
\hline $\mathrm{CR}$ & 161 & $1(0.62)$ & & & & \\
\hline $\mathrm{nCR}$ & 297 & $49(14.16)$ & & & & \\
\hline
\end{tabular}

HM: hematological malignancy; AL: acute leukemia; MDS: myelodysplastic syndrome; sAA: severe aplastic anemia; MM: multiple myeloma; HSCT: hematopoietic stem cell transplantation; CR: complete remission; nCR: no complete remission; AB: Acinetobacter baumannii.

\section{Figures}




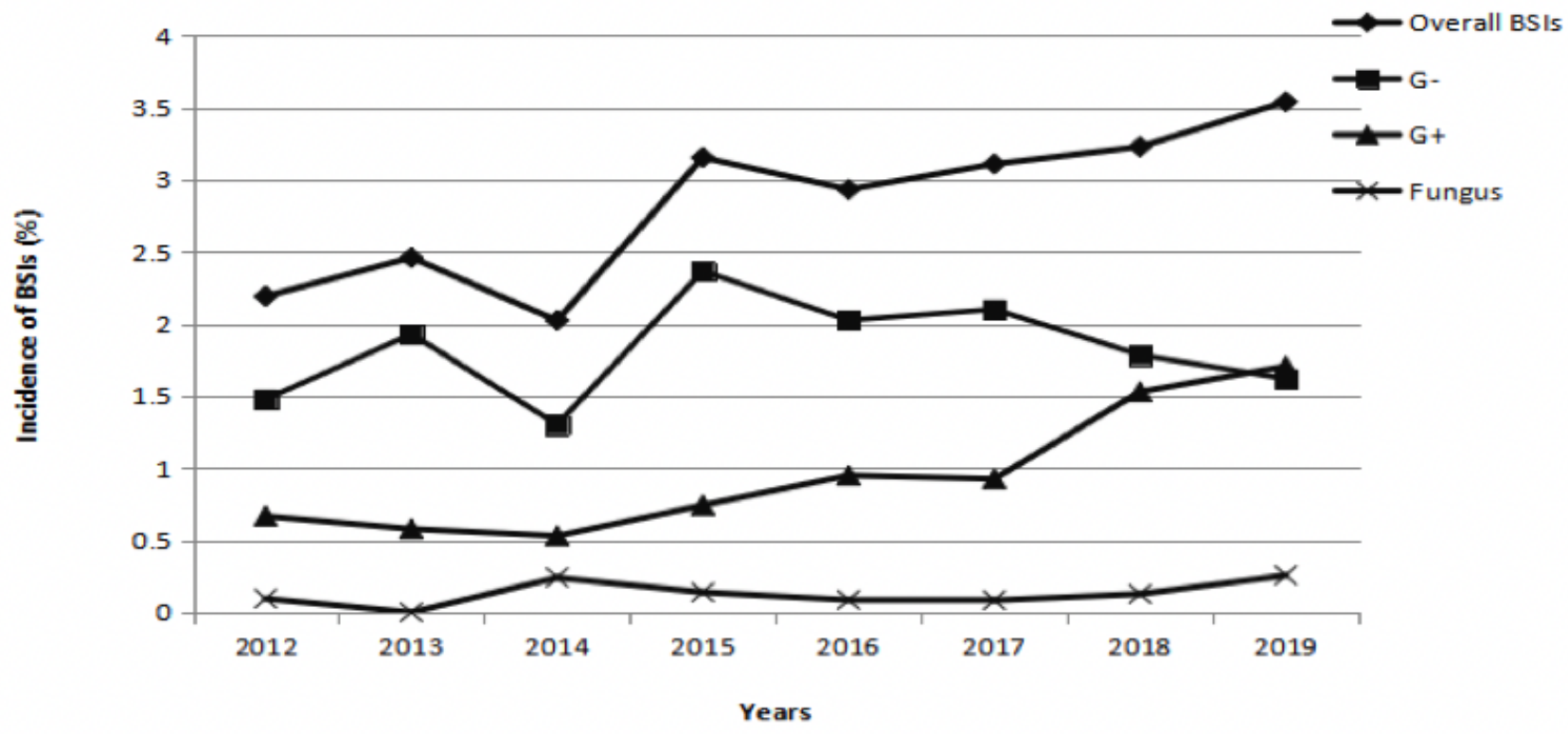

Figure 1

Trends in BSI pathogens among patients with hematologic malignancies in 2012-2019

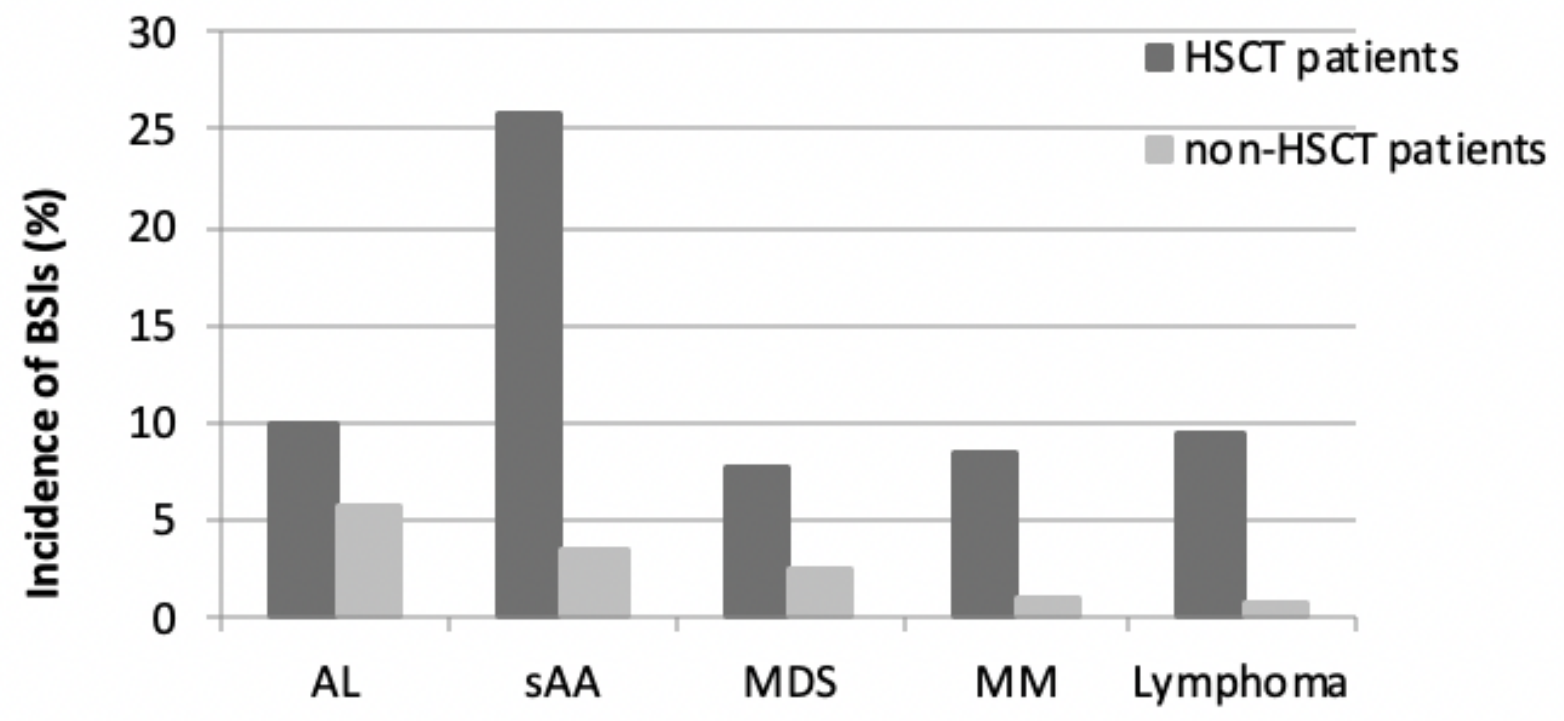

Figure 2 
Incidence of BSIs in HSCT and non-HSCT patients with different HMs. AL: acute leukemia; SAA: severe aplastic anemia; MDS: myelodysplastic syndrome; MM: multiple myeloma. The incidence of BSIs was significantly higher in the HSCT group than in the non-HSCT group for all kinds of HM. AL: $\chi 2=11.046, P$ $=0.001 ;$ sAA: $\chi 2=11.969, P=0.001 ;$ MDS: $\chi 2=4.712, P=0.030 ; M M: \chi 2=17.140, P=0.000 ;$ lymphoma: $\chi 2=127.782, P=0.000$. 